ÉGYPTE

monde arabe

\section{Égypte/Monde arabe}

12-13 | 1993

Une économie en transition

\title{
Le financement des services sociaux du secteur associatif à référent islamique en Égypte
}

Éléments d'information

\section{Sarah Ben Néfissa}

\section{(2) OpenEdition}

\section{Journals}

Édition électronique

URL : https://journals.openedition.org/ema/1270

DOI : 10.4000/ema. 1270

ISSN : 2090-7273

Éditeur

CEDEJ - Centre d'études et de documentation économiques juridiques et sociales

Édition imprimée

Date de publication : 31 mars 1993

Pagination : 213-239

ISSN : 1110-5097

Référence électronique

Sarah Ben Néfissa, "Le financement des services sociaux du secteur associatif à référent islamique en Égypte », Égypte/Monde arabe [En ligne], 12-13 | 1993, mis en ligne le 08 juillet 2008, consulté le 07 juillet 2022. URL : http://journals.openedition.org/ema/1270 ; DOI : https://doi.org/10.4000/ema.1270

Ce document a été généré automatiquement le 7 juillet 2022

Tous droits réservés 


\title{
Le financement des services sociaux du secteur associatif à référent islamique en Égypte
}

Éléments d'information

\author{
Sarah Ben Néfissa
}

1 Aborder la question du financement des services sociaux islamiques peut sembler un exercice périlleux tant sont lourds les préjugés et fantasmes qu'elle suscite. Deux principaux «lieux communs » apparemment contradictoires ont généralement cours sur cette question. Selon le premier, «l'argent vient d'ailleurs » : le financement, plus ou moins occulte, proviendrait des pays du Golfe; selon le deuxième, ces services sociaux ou caritatifs ne seraient pas véritablement dénués d'intérêt pour leurs gestionnaires : ils seraient source de profit, matériel ou autre (intérêts politiques, de prestige, etc.), de manière plus ou moins directe ou immédiate.

2 Ces préjugés ne sont pas totalement dénués de fondement. Un document publié en 1983 par l'Union générale des associations et fondations privées ${ }^{1}$ consacre un article à la question du financement des services sociaux du secteur associatif. L'auteur ${ }^{2}$, directeur de l'administration centrale de l'Assistance sociale au ministère des Affaires sociales, y décrit les différentes sources de financement autorisées par la loi. Dans la partie consacrée aux dons et testaments effectués au profit des associations et provenant d'Égypte ou de l'étranger, il constate que «les dons en provenance de l'étranger ont beaucoup augmentés du fait de la participation des travailleurs égyptiens à l'étranger et des frères arabes au soutien des projets sociaux ?. Toutefois, précise-t-il,

« l'autorisation du ministère des Affaires sociales est requise pour recevoir des dons de l'étranger. Ainsi le ministère, informé de la tournure indésirable qu'avaient pris les contacts de certaines associations avec des notables du Golfe pour obtenir des dons, a émis une circulaire administrative sur la manière dont il comptait orienter les dons en provenance de l'étranger.... »

3 De même, on peut noter sans exagérer qu'à toute action caritative viennent se greffer des enjeux multiples - psychologiques, économiques ou politiques au sens large. Un tel constat ne diminue en rien la valeur ou l'efficacité de l'action elle-même, d'autant que 
le phénomène n'est pas propre à l'activité sociale islamique (écartons toutefois les exemples caricaturaux d'action de type " religieux » à finalité clairement commerciale, comme les associations de hajj et de ziara qui pullulent en Égypte depuis le début des années 1980).

4 L'objectif du présent article n'est pas de lever la totalité des préjugés qui ont généralement cours sur le financement des activités sociales islamiques, mais de montrer que la question est plus complexe qu'il n'y paraît. En réalité, plusieurs systèmes de financement coexistent, de l'aide des pouvoirs publics à celle de grandes institutions - islamiques ou non - jusqu'à l'autofinancement que permet une gestion efficace des services rendus, en passant par la petite entraide de quartier. Il s'agit également de montrer qu'il existe un financement islamique interne à l'Égypte et que les actions caritatives qu'il soutient, loin d'être des pratiques «à la petite semaine», peuvent drainer des sommes importantes.

5 Nous décrirons également le dispositif institutionnel prévu par les pouvoirs publics pour le financement de l'action sociale privée et nous examinerons comment le mouvement caritatif islamique le met en application; en nous basant sur les rapports du ministère des Affaires sociales, les données du CAPMAS, les études effectuées sur la question et la présentation de quelques cas exemplaires de financement. Enfin, nous émettrons quelques hypothèses sur l'attitude contradictoire des pouvoirs publics envers les associations à référent islamique, à partir des éléments fournis sur la manière dont les autorités égyptiennes essaient de contrôler les différents systèmes de financement permis par la loi.

6 Avant d'entrer dans le vif du sujet, nous tenons à avertir le lecteur des limites que nous avons parfois rencontrées dans notre entreprise, telles que la rétention de l'information dans le domaine traité (tant de la part des pouvoirs publics que des associations ou des donateurs eux-mêmes), les contradictions entre les différentes sources (contradictions entre les chiffres, différences dans la présentation des données, nomenclature différente selon les sources, etc.), l'inadéquation entre les classifications des associations et les réalités de la vie associative, enfin la parution tardive des rapports officiels sur le sujet ${ }^{4}$.

\section{Le financement de l'action sociale privée}

7 Les pouvoirs publics égyptiens ne font pas de différence entre les services sociaux islamiques ou le financement islamique et les autres services sociaux ou financements privés : la législation concerne l'ensemble du secteur associatif et la loi n 32 de 1964 ne distingue que deux types de financement: le public et le privé. Le financement public consiste dans les différentes aides octroyées par la Caisse d'aide aux associations, fondations privées et unions d'associations.

\section{La Caisse d'aide aux associations et fondations privées, ou l'impossible médiation entre le financement privé et le secteur associatif}

8 Créée par la loi de 1964, cette Caisse a pour but de placer sous la responsabilité du ministère des Affaires sociales l'ensemble des sommes publiques ou privées destinées 
aux associations. Cette centralisation de la collecte et de la redistribution des fonds n'a rien de surprenant : elle correspond au renforcement du contrôle de la vie associative mis en place par la réforme de $1964^{5}$. Le conseil d'administration de la Caisse est principalement composé de responsables administratifs du ministère des Affaires sociales, d'un représentant du ministère des Waqfs (Biens religieux), d'un représentant du ministère de l'Administration locale et enfin de personnalités connues pour leur expérience dans le domaine des oeuvres sociales.

9 La Caisse a pour prérogatives de fixer les conditions d'octroi des aides et d'en organiser la distribution (aides à la création, à l'équipement, aides exceptionnelles, aides nouvelles, augmentation des aides régulières ou annuelles...). Enfin, elle fait parvenir aux directions des affaires sociales, dans les gouvernorats, une partie ${ }^{6}$ de ses ressources afin que celles-ci distribuent les aides annuelles aux associations concernées. La Caisse centrale dispose donc, dans 25 gouvernorats d'Égypte, de caisses régionales chargées de collaborer à la distribution et d'émettre des avis sur le travail que fournissent les associations subventionnées par l'État. Toutefois, les décisions importantes notamment l'octroi de nouvelles aides régulières - sont prises au niveau central et non régional.

La Caisse est elle-même alimentée par :

1. l'ensemble des taxes supplémentaires imposées au profit des oeuvres de charité par la loi $n^{\circ} 63$ de 1942 (entre autres, taxes sur le prix d'entrée dans différents lieux de spectacle, les tickets de chemin de fer et divers services ${ }^{7}$;

2. les sommes consacrées aux associations et fondations dans le budget de l'État ${ }^{8}$;

3. l'impôt sur les paris dans les jeux, régi par la loi n ${ }^{\circ} 10$ de 1922, dont une partie, consacrée aux structures s'occupant de la jeunesse, relève du ministère des Affaires sociales en accord avec celui de la Jeunesse ${ }^{9}$;

4. les sommes obtenues grâce aux tombolas du ministère des Affaires sociales ${ }^{10}$ (qui se montaient à 970.209 LE en 1990);

5. les dons régis par la loi $n^{\circ} 26$ de 1954 (art. 40 §) sur les sociétés par actions et les sociétés à responsabilité limitée, interdisant à celles-ci toute donation à des organisations ou partis politiques mais leur permettant de subventionner des actions à finalité sociale. Cette dernière source de financement est encore largement inexploitée alors qu'elle devrait être primordiale, de l'avis même des responsables administratifs ${ }^{11}$. Cela n'est pas surprenant et constitue même une des caractéristiques du financement des associations dans les pays en voie de développement ${ }^{12}$, contrairement à certains pays développés (États-Unis, par exemple) où le secteur privé finance pour une large part ce type d'organisations.

11 L'interventionnisme étatique, dans les pays arabes en général et en Égypte en particulier, est un des facteurs qui entravent la participation du secteur privé lucratif au financement des associations. La médiation de la Caisse d'aide aux associations et fondations privées ne contribue pas à encourager cette participation et certaines sociétés commerciales préfèrent verser leurs dons directement aux associations qu'elles connaissent et qui bénéficient de leur confiance, ou financer directement des œuvres caritatives en se passant de toute médiation. Cette donnée n'échappe pas aux responsables administratifs : le document précédemment cité ${ }^{13}$ émet le vœux que les associations entrent en contact avec les sociétés commerciales pour obtenir des financements, cela en attendant que la Caisse adopte des décisions mettant en application la loi précédemment citée; ces mêmes responsables notent que les obligations religieuses concernant les aumônes telles que la zakat et la sadaqa sont un 
facteur important d'encouragement aux dons. Apparemment, la Caisse ne parvient pas non plus à canaliser vers elles ces donations, bien que l'islamisation de la société égyptienne constatée à partir des années 1970 ait favorisé le renouveau de pratiques religieuses comme l'aumône. Les seules donations qui lui parviennent sont la zakat des banques islamiques existant en Égypte, mais le montant en est infime comparativement aux donations directes aux associations.

12 Les aides consenties par la Caisse sont de plusieurs types: aides cycliques, exceptionnelles, aides à la création et à l'équipement, aides provenant des budgets d'autres ministères, et pour finir, somme destinée à couvrir les salaires minimum des employés des associations. Les aides à la création constituent la part la plus importante des aides consenties, mais il importe de rappeler qu'une association, pour pouvoir en bénéficier, doit pourvoir à $50 \%$ minimum de ses dépenses de départ. Quant aux aides exceptionnelles, elles sont dispensées avec liberté, au contraire des aides cycliques qui deviennent une obligation pour le ministère une fois décidé leur octroi et à condition, évidemment, que les associations concernées réalisent véritablement leurs objectifs et respectent la loi. Ces aides servent à couvrir soit des dettes ou des déficits subits, soit les dépenses exceptionnelles que peut engendrer une activité nouvelle.

Les conditions essentielles exigées par la Caisse pour venir en aide aux associations sont les suivantes: en ce qui concerne les nouvelles aides cycliques ou l'augmentation de leur montant, il importe que les activités de l'association ne s'adressent pas à ses seuls membres. Il existe en effet en Égypte deux types d'associations, celles dont les services sont destinées à un large public et celles qui ne les fournissent qu'à leurs adhérents. Généralement, il s'agit d'associations d'employés d'administrations ou de sociétés comme l'Association des fonctionnaires du ministère de la Santé (sud du Caire) ou l'Association des employés de la Société du plastique (Giza). Il peut également s'agir d'associations de personnes originaires d'un même gouvernorat ou d'un même village, comme l'Association des personnes originaires du gouvernorat d'Assouan et installées à Alexandrie. Ces types d'associations fonctionnent comme des caisses d'épargnes offrant à leurs membres certains services ou avantages : voyages organisés à prix modique, prêts, etc. ${ }^{14}$. Il faut également que l'association ait au moins un an d'activité et que les services qu'elle propose répondent véritablement à un besoin local. L'aide qu'elle reçoit est limitée à un plafond de $75 \%$ des dépenses totales de l'année qui précède.

Quant aux aides de démarrage ou d'investissement, leur dispense est également soumise à certaines conditions: elles ne doivent pas être utilisées à la seule construction d'un local. Encore faut-il que ce local soit le support d'activités "nécessaires à l'environnement», selon la conception de la direction des Affaires sociales et en fonction de la vocation du ministère. Si tel est le cas, la condition imposée à l'association de disposer au départ d'un avoir de $50 \%$ peut être levée.

Les aides provenant d'autres ministères intéressés par les activités de certaines associations (Santé, Culture, Recherche scientifique, Waqfs, Affaires étrangères) sont d'abord versées à la Caisse centrale, à charge pour celle-ci de les redistribuer. Dans la pratique, la Caisse rencontre maintes difficultés pour effectuer cette redistribution: beaucoup d'associations ne respectent pas les obligations leur incombant, comme la convocation annuelle de l'assemblée générale ou la tenue rigoureuse de la comptabilité, état de choses qui retarde le distribution des aides. Du reste, l'Organisme central de la comptabilité nationale a émis de sévères critiques sur les finances de certaines associations. Mais cette situation n'est-elle pas compréhensible du fait de la multiplicité 
des règles bureaucratiques et tatillonnes imposées aux associations par la loi $\mathrm{n}^{\circ} 32$ de 1964 , et notamment par son décret d'application ${ }^{15}$ ?

Hormis ces aides, la Caisse consent également aux associations un crédit qui leur permettra d'appliquer la loi $n^{\circ} 64$ de 1974 sur le salaire minimum des employés du secteur privé. On constate à ce propos que les associations n'utilisent pas entièrement ce crédit ${ }^{16}$, bien qu'il ait été augmenté pour tenir compte des hausses successives de salaires des employés. Le phénomène est d'autant plus étonnant que selon une étude de la Ford Fondation ${ }^{17}$, le salaire des travailleurs du secteur privé a augmenté d'environ $65 \%$ au cours des cinq dernières années, outrepassant par conséquent les possibilités financières des associations. Cela s'explique probablement du fait que l'octroi de ce crédit est soumis à la déclaration obligatoire, par les associations, de la liste de leurs employés. Bon nombre d'entre elles s'en abstiennent et c'est peut-être là un des facteurs de leur équilibre budgétaire. A titre d'exemple, la majorité des médecins travaillant dans le secteur associatif déclarent des vacations et non des salaires.

\section{Les Banques islamiques : une zakat dérisoire}

17 Selon le rapport annuel de 1991 de la Banque Fayçal, 95,96 \% des ressources de sa caisse de la zakat, soit 4.863.281 de LE, ont été distribués aux personnes ou institutions pouvant bénéficier de la zakat, familles pauvres, étudiants, mosquées privées, concours de récitation du Coran, bibliothèques religieuses, associations médico-sanitaires. On peut constater que la somme versée à ces dernières a augmenté par rapport à l'année 1990 : de $492.241 \mathrm{LE}$, elle est passée à 1.219.181 LE ${ }^{18}$, soit 18,83\% du total des dépenses de la caisse de la zakat de 1991. Le rapport précise par ailleurs que les dispensaires recevant une aide financière sont ceux rattachés à des mosquées dépendant du ministère des Waqfs.

La Banque internationale islamique d'investissement et de développement a également une caisse de la zakat et son montant, comme le signale son rapport 1990, s'élevait à 63.000 LE, dont seulement 13.000 ont été consacrées à 15 institutions, mosquées et associations de charité, le reste étant versé à 893 cas sociaux bénéficiaires de la zakat. Le rapport signale encore que le restant de la zakat de 1989 (574.000 LE) et son placement contribuent également à aider des nécessiteux.

Quant à la Banque saoudo-égyptienne, son rapport annuel ne signale aucun versement de zakat sous quelque forme que ce soit, ceci bien que cet établissement se proclame "islamique». Les banques ordinaires ayant des filiales islamiques ne versent pas de zakat en tant qu'institutions, mais certains de leurs clients leur demandent de retirer une partie de leur avoir en tant que zakat et de la redistribuer. Il est important de noter ici que ces institutions financières islamiques, tout en cherchant à avoir de bons rapports avec l'État égyptien en lui déléguant la distribution d'une partie de leur zakat, préfèrent gérer elles-mêmes directement leurs pratiques caritatives. Cela leur permet de ménager leur image de marque islamique et d'apparaître clairement aux yeux du public comme pourvoyeurs de fonds à destination charitable.

20 Les rapports de la Banque paraétatique Nasser Ijtimâ'i ${ }^{19}$, qui parraine les 4.500 comités de la zakat, ne signalent pas de versements de subventions aux associations, mais les rapports financiers de certaines associations ${ }^{20}$ à référent islamique précisent qu'elles reçoivent des dons de part de la Banque Nasser, situation qui s'explique probablement par le fait que cette dernière concurrence les associations caritatives islamiques 
indépendantes de l'État, opère par conséquent un choix «politique » des associations qu'elle subventionne et préfère, par prudence, gérer directement ses activités sociales, notamment par l'intermédiaire de son réseau de comités qu'animent près de 31.500 personnes.

Le volume de l'aide des institutions bancaires existant en Égypte et passant par la médiation de la Caisse semble dérisoire par rapport aux aides versées directement aux associations par certaines sociétés ou par certains hommes d'affaires égyptiens. La presse égyptienne est riche d'informations à ce propos. Ainsi le quotidien AlGumhuriya ${ }^{21}$ annonce-t-il que "six nouveaux complexes islamiques vont être créés dans la ville de Manzala à partir de donations privées d'une valeur de 17.000 LE. » De même, selon le quotidien Al-Akhbar ${ }^{22}$, "500.000 LE ont été rassemblées par les citoyens d'un village du gouvernorat d'Assiout pour construire une mosquée, un institut religieux et une salle de cérémonies. » Enfin, il est intéressant de noter la grande disparité des sommes versées à titre de zakat à l'association «La nuit du Destin», fondée par le journaliste bien connu Mustapha Amin (d'al-Akhbar), qui peuvent en effet s'échelonner ${ }^{23}$ de 5 à 30.000 LE $^{24}$.

\section{Les différentes formes de l'aide publique}

\section{La problématique nomenclature des associations}

Les types d'associations privilégiées par l'aide publique en termes quantitatifs sont difficiles à repérer du fait de la différence de nomenclatures d'une source à l'autre. D'une part, les textes juridiques définissent 14 domaines d'activités associatives ${ }^{25}$ mais permettent aux associations d'oeuvrer dans plus d'un domaine après autorisation de l'administration. D'autre part, les associations qui ont choisi le «développement social » comme domaine d'activité sont celles qui ont le plus de liberté d'action; elles couvrent en fait une multitude d'activités, notamment dans le domaine économique. Enfin - et de l'avis même des responsables -, la nomenclature prévue par la loi ne correspond plus véritablement aux réalités de la vie associative et une nouvelle nomenclature devrait être proposée ${ }^{26}$. Ainsi, certaines activités n'ont que peu de succès dans les choix opérés par les fondateurs d'associations (par exemple, «les relations entre le peuple égyptien et les peuples amis » ou «l'aide aux prisonniers et à leurs familles »...).

Cette inadéquation entre les textes et la réalité a pour conséquence de légitimer les libertés que prennent les différentes instances administratives avec la nomenclature officielle des associations. Ainsi le ministère des Affaires Sociales ne donne d'informations précises que sur deux grandes catégories d'associations, celles dites « de développement social» et celles qu'il appelle "d'assistance sociale", catégorie "fourre-tout» regroupant toutes les associations qui ne font pas de développement social. De plus, ce même ministère ne mentionne plus les associations à caractère religieux, bien qu'elles existent toujours et sur le plan juridique et dans la réalité. Il se cantonne à parler prudemment d'activités "culturelles" et "scientifiques». Le $\mathrm{CAPMAS}^{27}$ opère également une catégorisation qui lui est propre, plus détaillée que celle du ministère des Affaires sociales. Sa nomenclature repose sur 6 domaines d'activités : enfance et maternité, famille, aides sociales à la vieillesse et aux familles de prisonniers, aides à des catégories particulières de population comme les handicapés, 
activités culturelles, scientifiques et religieuses, enfin associations qui embrassent plus d'un domaine d'activité.

24 Le problème principal des informations du CAPMAS est leur ancienneté $(1986,1987$, $1988)^{28}$ et leur limitation aux seules associations ayant communiqué des informations sur leurs activités (c'est le cas de 1.084 d'entre elles pour le dernier rapport publié en 1992).

\section{La faiblesse du volume de l'aide publique}

Sur la totalité des 12.832 associations existant en Égypte en $1989 / 90^{29}$, seules 4.448 (soit $34,66 \%)$ d'entre elles reçoivent des aides de la part de l'État. La part la plus importante $(31,39 \%)$ est fournie par le ministère des Affaires sociales, qui a octroyé en 1990 à 4.028 associations (2.043 d'assistance sociale et 1.985 de développement social) une enveloppe globale de 14.009.082 LE (soit une moyenne de 3.477,92 LE par association). Les autres ministères ont soutenu financièrement 420 associations ( 318 d'assistance sociale et 102 de développement social) avec une enveloppe globale de $1.732 .644 \mathrm{LE}$ (soit une moyenne de 4.153,42 LE par association).

\section{a) Répartition de l'aide selon le type d'activité}

Le dernier rapport du CAPMAS ne concerne, comme on l'a vu plus haut, que 1.084 associations dont 230 , soit $21,27 \%$, ont des activités de type religieux (sans qu'il soit précisé si elles sont musulmanes ou chrétiennes), culturel ou scientifique ${ }^{30}$. Le volume de l'aide publique octroyée à ces dernières est de 956.959 LE, alors que l'État accorde aux 174 associations qui s'occupent de la maternité et de l'enfance la somme globale de 1.261.840 LE, et 779.692 LE aux 259 associations d'aide sociale. Si les associations de type religieux ne sont pas écartées de l'aide publique, elles ne semblent pas non plus privilégiées. Il faut toutefois nuancer cette affirmation : selon le document du CAPMAS en effet, les pouvoirs publics octroient 2.832.518 LE aux 241 associations qui embrassent plus d'un domaine d'activité. Or la pluralité des activités est une des caractéristiques du mouvement associatif musulman, selon la dernière étude approfondie effectuée sur la question $^{31}$. De plus, selon le même document, le coût des activités "culturelles, scientifiques et religieuses » de cette catégorie d'association, qui s'élève à 2.661.828 LE, est supérieur au coût des autres types d'activité comme les "aides sociales" (1.886.162 LE) ou la «famille» (1.586.757 LE). Aussi peut-on avancer que certaines associations à réfèrent islamique semblent favorisées par les pouvoirs publics.

\section{b) Les associations d'intérêt général}

Toutefois, le versement de subventions n'est pas la seule manière dont l'État égyptien privilégie certaines associations. Il en existe d'autres, notamment leur déclaration au Journal officiel comme étant "d'intérêt général »: les associations bénéficiant d'un tel statut sont privilégiées par le ministère en ce qu'il les considère comme faisant pratiquement partie de son administration. Leurs biens ne peuvent être confisqués, elles ne peuvent être expropriées par l'État. Actuellement, sur un total de 330 associations déclarées d'intérêt général, 128 - soit 38,78\% - ont un référent islamique (parmi les plus importante, citons l'Association de sauvegarde du Coran, l'Association des jeunes filles musulmanes, l'Association islamique de services sociaux, l'Association 
des femmes islamiques, l'Association de charité islamique, etc., sans oublier les grandes associations disposant de filiales sur tout le territoire comme la Gama'iyya Cha'riyya, l'Association des Ansars de la sunna muhammadiyya, l'Association des jeunes musulmans, etc.).

\section{c) L'aide du ministère des Waqfs}

Aux aides octroyées par le ministère des Affaires sociales s'ajoutent celles que dispense le ministère des Waqfs. Or, le rapport ${ }^{32}$ établi par ce dernier sur ses activités caritatives montre qu'il ne soutient que deux associations, bien connues en Égypte, l'Association des jeunes musulmans et celle des jeunes filles musulmanes, alors que le nombre des associations à référent islamique dépasse 3.500 au bas $\operatorname{mot}^{33}$. Cette aide, il est vrai, est importante: les deux associations ont reçu globalement $65.000 \mathrm{LE}$ pour l'année 1988/89, 60.000 LE pour l'année 1989/90 et 60.000 LE également pour l'année 1990/91. Rappelons ici que l'Association des jeunes musulmans est très ancienne et rayonne sur tout le territoire égyptien avec plus d'une centaine de filiales.

Le ministère des Waqfs n'a donc sur le mouvement associatif musulman qu'une emprise très limitée, à la différence du ministère des Affaires sociales et, surtout, de la Banque Nasser Ijtimâ'i qui apparaît comme un des piliers de l'islam « social » officiel égyptien ${ }^{34}$. L'analyse du rapport du ministère des Waqfs sur ses activités caritatives le confirme: plus de $80 \%$ de la somme consacrée à ces activités sont en fait destinés aux fonctionnaires du ministère eux-mêmes. Ainsi, sur les 17 postes de dépenses énumérés dans le rapport, 8 sont des bourses mensuelles pour étudiants fils de fonctionnaires ou des aides temporaires destinées à des fonctionnaires à l'occasion de leur mariage ou du mariage de leurs enfants, ou à l'occasion de leurs funérailles, de leurs maladies, ainsi que des aides temporaires pour les membres des commissions, des versements pour les employés des commissions, enfin des recouvrements de prêts pour fonctionnaires décédés.

Ces données expliquent en partie le discrédit dont pâtit le ministère des Waqfs, dont une des principales vocations est pourtant la bienfaisance à partir du revenu des biens religieux. Elles expliquent également l'intitulé d'un article paru dans le journal Al-Liwâ al-islâmi $i^{35}$, "Où va l'argent de la bienfaisance ? ", dont l'auteur interroge à ce propos la responsable de l'administration de la bienfaisance au ministère des Waqfs. Celle-ci constate tout d'abord la diminution des biens religieux, par conséquent celle de leurs revenus. " Aujourd'hui, dit-elle, il est possible de compter ces biens sur les doigts d'une main ». A une question concernant les critères de distribution aux nécessiteux du budget de l'administration de la bienfaisance, la responsable déclare que «cette administration rencontre parfois des situations exceptionnelles et catastrophiques. Par exemple, s'il arrive qu'un des wakîl (délégués) du ministère est subitement atteint d'une maladie rénale, je ne vois pas d'inconvénient à lui fournir une aide pour qu'il se soigne. Or il ne faut pas dire que j'ai aidé un wakîl du ministère, mais un malade souffrant d'insuffisance rénale !» 


\section{L'autofinancement : une réussite relative pour les associations a référent islamique}

\section{Les « dons spontanés »}

31 L'autofinancement, deuxième grande catégorie de financement de l'action associative, peut revêtir plusieurs formes : acquittement d'un droit d'adhésion à l'association ${ }^{36}$ ou «dons spontanés", comme le ministère des Affaires sociales les dénomme, sommes versées par le public aux associations qui ont gagné sa confiance. Cela se manifeste notamment lors des fêtes religieuses ou, comme il a été mentionné plus haut, au moment des versements rituels des deux types de zakat imposées par l'islam.

Il n'est pas donc pas étonnant que ce soit les associations à réfèrent religieux qui en bénéficient principalement, d'autant que le législateur (art. 17 du décret d'application de la loi n ${ }^{\circ} 32$ de 1964) ne considère comme " dons spontanés ", c'est-à-dire non soumis au contrôle des autorités administratives, que les dons effectués dans les lieux de culte. Or la première caractéristique d'une association à référent islamique est de disposer d'une mosquée à l'intérieur de ses bâtiments. En effet, selon les statistiques du CAPMAS de 1992, les associations qui reçoivent le plus de dons sont celles qui gèrent plus d'une activité $^{37}$, ce qui est principalement le cas, selon nos déductions antécédentes, des associations à référent musulman.

33 Ainsi, pour l'année 1988, les 241 associations de ce type ont reçu une somme totale de 2.241.101 LE sous forme de dons, précédant de loin, de ce point de vue, les autres types d'associations. Notons ici que les sommes obtenues par les caisses de la zakat patronnées la Banque Nasser ljtimâ'i - caisses qui se trouvent parfois dans des locaux d'associations - ne sont pas versées au budget de ces associations mais rassemblées par la Caisse centrale de la zakat de la Banque, qui se charge elle-même de les reverser à des œuvres caritatives ou sociales. Selon les principes affichés par la Banque Nasser, ce reversement se fait au prorata de la somme que chaque caisse locale est parvenue à collecter; il en va différemment en réalité et il est significatif de noter les réserves émises sur ce point par le responsable de l'administration centrale de l'aide sociale au ministère des Affaires sociales ${ }^{38}$ : «Le ministère a constaté que les comités de la zakat qui collectent de l'argent dans les mosquées dépendant des associations sont extérieurs aux activités de ces associations. Il est nécessaire de remédier à cette situation par l'instauration de règles adéquates, afin que la collecte de la zakat soit effectuée par l'association elle-même, au profit de ses propres activités et au profit de l'intérêt général... »

\section{Les collectes soumises à autorisation ministérielle}

34 C'est là une autre grande source d'autofinancement. Chaque association n'a droit qu'à deux autorisations par an. En 1989/90, le ministère des Affaires sociales a accordé aux associations d'assistance sociale 481 autorisations de collecte (ce qui a représenté 21.602.191 LE), et 64 aux associations de développement social (soit 3.365.737 LE).

Les collectes sont surtout utilisées pour financer des associations anciennes, qui ont pignon sur rue, comme l'Association d'entraide hivernale, ou l'Association d'amélioration de la santé, ou l'Association d'aide aux étudiants. Toutefois, certaines associations locales ont eu recours à la collecte pour financer quelques-uns de leurs 
importants projets. La réussite de ce mode de financement dépend largement des personnalités qui animent l'association, de leur position sociale (médecins renommés, députés de l'Assemblée du peuple, riches commerçants, etc.) et surtout de leur réputation d'honnêteté. Les relations humaines ont une importance considérable dans ce type d'opération, de même que la clarté des objectifs de la collecte. Il n'est pas étonnant, dès lors, que le ministère opère un contrôle important avant d'accorder les autorisations, et les soumet à des conditions draconiennes. Toutefois, la volonté de protéger le public d'éventuelles escroqueries n'explique pas entièrement le faible nombre d'autorisations accordées annuellement, nombre dérisoire par rapport à celui des associations existant en Égypte (même si l'on sait qu'au moins 50 \% d'entre elles n'ont pas d'activité réelle) ${ }^{39}$. Sans doute s'ajoute-t-il à cette motivation une sélection d'ordre politique.

Les collectes peuvent prendre plusieurs formes: ventes de timbres-poste, réception d'argent contre reçu ou dans des caisses prévues à cet effet, fêtes de charité ou ventes de charité. Pour donner un exemple concret de l'esprit qui préside à ce genre de collectes, citons ici certains passages du prospectus distribué par l'Association médicale islamique dans le but de rassembler les fonds nécessaires à la construction d'un hôpital. Le texte commence par « souhaiter les meilleurs vœux à la communauté musulmane à l'occasion de l'ayd al-fitr " puis annonce, immédiatement après, "l'intention de l'association de fonder à Héliopolis un hôpital "islamique" de 350 lits, doté d'une section de chirurgie et de radiologie, de laboratoires, d'une pharmacie, d'un service de stérilisation central», etc. Le prospectus précise ensuite que l'hôpital soignera gratuitement ceux qui ne pourront s'acquitter des soins, lesquels s'adresseront « aussi bien aux musulmans qu'aux non musulmans ». Puis il énumère les étapes déjà franchies dans la réalisation du projet: don d'un terrain de plus de $20.000 \mathrm{~m} 2$ par le ministère de la Défense, établissement du plan de l'hôpital, début des travaux. Le coût du projet est estimé à 21 millions de LE, et le prospectus précise que les donations aux hôpitaux font partie des destinations légitimes (d'un point de vue religieux) de la zakat, selon les avis des ulémas. A la fin du prospectus sont énumérées les adresses auxquelles les mandats peuvent être envoyés (Banque islamique Fayçal, une des filiales de la Banque Misr, une des filiales de la Banque Ahli ainsi que les noms des médecins responsables de l'Association médicale islamique).

Les chiffres du CAPMAS montrent que ce sont les 241 associations qui embrassent plus d'un domaine d'activité et les 259 associations d'aide sociale qui collectent par ce moyen les sommes les plus importantes : 1.879.068 LE et 2.541.533 LE respectivement pour l'année 1988.

\section{Dons de l'étranger ou d'institutions étrangères installées en Égypte}

La loi permet également aux associations de recevoir des dons de l'étranger. La circulaire administrative émise en 1983, destinée à juguler les dons provenant des pays du Golfe selon les critères du ministère, n'a apparemment pas suffi puisque le nouveau code des douanes promulgué en 1986 a remis en cause les exonérations douanières sur les fonds versés par l'étranger au profit du secteur associatif. Cette réforme a conduit les donateurs étrangers à ne faire que des dons en nature, et selon la liste limitative imposée par la loi $n^{\circ} 260$ de l'année 1984 réformée par la loi de 1986,12 catégories de dons en nature sont exemptés de taxes douanières (appareils et fournitures nécessaires 
aux mosquées dépendant d'associations, refroidisseurs d'eau, ventilateurs de plafond, microphones, aspirateurs, moquettes, tapis...).

Les dons de l'étranger ont pour première provenance des pays musulmans, pays du Golfe surtout quoique non exclusivement. Plusieurs associations reçoivent des dons de pays occidentaux (notamment de pays où vivent d'importantes communautés égyptiennes). Ainsi les activités de l'Association Bamby pour la mère et l'enfant, installée à Alexandrie, sont financées à plus de $75 \%$ par les donations d'égyptiens d'Alexandrie installés au Canada.

Les associations reçoivent également des subventions de la part d'institutions étrangères ou internationales installées en Égypte. Si la sélection opérée par les donateurs n'est pas dénuée d'arrière-pensées politiques, il n'en demeure pas moins qu'un de leurs principaux critères est la réussite sur le terrain et l'ancrage dans l'environnement des associations choisies. Les associations à réfèrent islamique, notamment celles dont les animateurs sont proches des pouvoirs publics, ne sont pas écartées de l'aide internationale (c'est le cas de l'Association de Sidi Gaber et de l'Association de Sidi Bichr, installées à Alexandrie, qui ont reçu des dons en nature et en espèces de l'UNICEF et de l'UNESCO).

\section{Revenus tirés des services et produits offerts par l'association}

41 C'est la plus intéressante des formes d'autofinancement. C'est à ce niveau en effet qu'il est possible de parier d'autofinancement au sens propre du terme : il s'agit là des fruits des activités propres de l'association, ce qui lui permet de ne pas être tributaire des aléas de l'aide publique ou même de l'aide privée. Les services les plus classiques fournis par le secteur associatif sont les garderies d'enfants, les hospices de vieillards, les foyers d'étudiants, les dispensaires et les hôpitaux, les centres de formation professionnelle, les ateliers, les écoles de langues, les cours particuliers pour les écoliers, etc. A titre d'exemple, citons les différentes activités de ce type organisées dans certains gouvernorats. Dans le gouvernorat de Minya, le secteur associatif parraine 33 ateliers de jeunes filles ou femmes, 10 ateliers de fabrication de tapis, 4 ateliers de menuiserie, 3 exploitations agricoles, un atelier de fabrication de miel, 1 boulangerie, un atelier de repassage, etc. Quant aux services sociaux offerts en échange d'une contribution financière, les plus importants - toujours dans le gouvernorat de Minya - se constituent de 70 garderies d'enfants, 12 cours particuliers, 5 clubs pour enfants, 11 cours d'alphabétisation, 2 laboratoires d'analyses, 13 bibliothèques, 26 dispensaires, 6 écoles, 1 pharmacie, 1 centre de formation professionnelle, un hospice de vieillards ; 11 associations offrent également des services funéraires, etc.

Dans le gouvernorat de Menufiyya, les associations parrainent notamment 6 lieux d'accueil pour migrants, 303 garderies d'enfants, 55 dispensaires, etc. Comme activités de type économique, il faut citer 18 ateliers de couture, 51 ateliers de fabrication de tapis, 19 ateliers de fabrication de miel, 16 ateliers de menuiserie, etc.

La majorité des services sont offerts en échange d'une somme inférieure à leur prix de revient et la plupart des associations les proposent gratuitement aux personnes démunies. Ainsi la moyenne des prix des garderies d'enfants du secteur associatif, sur l'ensemble du territoire, n'atteint pas $44 \%$ de leur coût réel ${ }^{40}$. Les associations arrivent à rééquilibrer leurs finances en tablant sur la quantité d'utilisateurs intéressés par la modicité des prix pratiqués. Il n'est donc pas étonnant que les services les plus porteurs 
et les plus attractifs soient ceux qui pallient les déficiences de l'État, notamment dans le secteur sanitaire et dans le secteur éducatif. Ainsi, alors que le nombre de femmes qui travaillent s'élève à $6.121 .000^{41}$ et le nombre d'enfants entre 4 et 6 ans à $3.000 .000^{42}$, les écoles publiques n'offrent en 1992 que 1.207 garderies d'enfants, laissant ainsi la voie libre aux garderies du secteur scolaire privé. Etant donné l'importance des besoins, les garderies sont devenues une activité lucrative intéressante pour ce dernier, et nombre d'entre elles ont été créées sans respect des règles sanitaires, architecturales et éducatives imposées par la loi n 50 de 1977. Or, en 1982, le secteur associatif gérait déjà 2.102 garderies.

Dans le domaine sanitaire également, le secteur associatif est parvenu à occuper la place laissée vacante par les carences du secteur public hospitalier (mauvais accueil, manque de moyens, manque de lits, personnel sous-payé, etc.) et la cherté du secteur privé. Le secteur associatif a joué sur la modicité des prix pratiqués et le chômage des médecins, dont les honoraires, dans ces associations, sont à mi-chemin entre ceux du privé lucratif et ceux du public. Ce sont généralement de jeunes médecins qui considèrent leur passage dans le secteur associatif comme une période de stage leur permettant de se constituer une clientèle, d'où une rotation importante. Les associations dispensent des soins de niveau moyen eu égard au personnel ou aux équipements dont elles disposent, à quelques exceptions près. Cependant, les malades $\mathrm{y}$ sont bien accueillis malgré une capacité d'accueil moindre que celle du secteur public (délais d'attente plus courts par exemple) et le prix de la consultation, s'il est supérieur à celui pratiqué dans les hôpitaux publics, est nettement inférieur à ceux du privé lucratif : il s'échelonne de 50 piastres à $1 \mathrm{LE}$ et les petites opérations chirurgicales ne dépassent pas $10 \mathrm{LE}$, alors que les médecins privés demandent entre 20 et $50 \mathrm{LE}$ pour de simples consultations. Les nécessiteux reçoivent des soins gratuits après enquête sociale. Il s'agit donc d'une médecine de masse peu sophistiquée.

Les associations parviennent malgré tout à équilibrer leurs finances du fait du grand nombre de demandeurs et de l'exemption fiscale sur les revenus dont elles bénéficient, exemption qui constitue leur principal atout par rapport au secteur médical privé. La majorité des médecins ${ }^{43}$ travaillant dans le secteur associatif sont également exemptés car ils déclarent des vacations et non des honoraires dans la plupart des $\operatorname{cas}^{44}$. A titre d'exemple, le secteur associatif parraine 23 hôpitaux et dispensaires dans le gouvernorat de Minya, 57 dans celui de Menufiyya et 7 dans celui de Damiette. Quant au gouvernorat du Caire, on y dénombre 10 hôpitaux et dispensaires dans le quartier de Matariyya, 11 dans le Vieux-Caire et 29 à Maadi.

Notons toutefois que ces dernières années, nombre d'observateurs ${ }^{45}$ ont constaté une élévation du prix des services offerts par le secteur associatif. Il faut probablement relier ce phénomène à la remise en cause des exonérations douanières dont il a été question plus haut, et également à la suppression par les pouvoirs publics, toujours en 1986, de la réduction de $50 \%$ des prix de l'électricité et du gaz consommés par les associations.

De l'avis des responsables administratifs ${ }^{46}$ et selon la dernière étude approfondie effectuée sur la question ${ }^{47}$, ce sont les associations religieuses chrétiennes ou islamiques qui réussissent le mieux sur ce terrain. Les données de 1988 fournies par le CAPMAS en 1992, portant sur les rentrées financières des 1.084 associations répertoriées dans le document, le confirment largement. Ainsi, dans la rubrique "rentrées d'argent en échange de services ", les associations qui gèrent plus d'une 
activité etdont la majorité sont à référent islamique, ont réussi à rassembler la somme de 6.695.806 LE, suivies de loin par les associations d'aide sociale avec 4.082.692 LE et celles de l'enfance et de la maternité avec 3.345.286 LE.

Il en est de même dans la rubrique "vente de produits ", où la réussite des associations à référent islamique est également manifeste. Elles arrivent ainsi en tête avec les associations d'aide à la famille, les sommes rassemblées se montant à 1.742.136 LE et 1.754.358 LE respectivement. Ce constat est encore plus significatif lorsqu'on sait que les associations d'aide à la famille bénéficient d'un soutien particulier de l'État qui, dans le cadre des programmes de "famille productive », leur fournit gratuitement des moyens de production, machines à coudre ou à tricoter par exemple.

\section{Revenus immobiliers, intérêts des participations et des certificats d'investissement}

49 L'article 8 de la loi $n^{\circ} 32$ de 1964 accorde aux associations des droits de propriété sur des immeubles à condition que ces propriétés soient en rapport avec les activités de chaque association. Toutefois, le même article exempte de cette dernière condition les associations travaillant dans le domaine social, plus précisément celles qui offrent des services médicaux ou une assistance aux familles, aux mères et aux enfants, ainsi qu'aux délinquants et aux personnes âgées incapables de subvenir à leurs besoins. Là encore, les associations à réfèrent islamique arrivent en tête par l'importance de leurs revenus. Selon le CAPMAS, elles rassemblent 2.009.558 LE, dépassant de loin les autres types d'associations de ce point de vue. Il s'agit donc d'associations riches qui disposent de sources de revenus stables leur permettant de faire face aux aléas d'autres types de ressources comme les dons ou même les revenus de leurs propres activités. Ce type de revenus a évidemment augmenté avec l'élévation des taux d'intérêts des dépôts bancaires, et il existe toujours à ce propos une controverse entre le trésorier de l'association et les membres actifs quant à l'utilisation de ces dépôts. Des campagnes de presse attaquent régulièrement ce genre de pratiques. Ainsi le quotidien gouvernemental Al-Gumhuriya du 19 juillet 1992 dénonce-t-il «les 5.000 associations religieuses qui ont oublié de lutter contre l'extrémisme et se consacrent à l'exposition de marchandises et aux excursions de la Umra (...) ; certaines sont devenues des sociétés à but lucratif pour lesquelles la fin justifie les moyens; d'autres ont oublié leur vocation pour louer leur siège principal comme salle d'exposition permanente de commercialisation de produits du bâtiment. D'autres ne s'occupent plus que de Hajj et de Umra (grand et petit pèlerinage), devenus de nos jours un commerce prospère - on ne compte plus les exemples à ce propos (....). Ainsi l'association des jeunes musulmans gagne de l'argent en louant son siège principal, de même qu'elle a loué son unique théâtre, qui servait de salle de conférences, à une société d'informatique... »

50 Au terme de cette étude, un certain nombre de constats peuvent être posés. Mais auparavant, il importe d'attirer l'attention du lecteur sur deux points concernant les sources utilisées. D'une part, les données fournies par le CAPMAS ne donnent d'informations que sur les associations ayant besoin de l'aide des pouvoirs publics et qui, par conséquent, leur rendent compte de l'état de leurs finances et de leurs activités. Ces associations font donc très probablement partie du réseau associatif islamique contrôlé sur tous les plans par le ministère des Affaires sociales, d'autant qu'on sait que les principales décisions d'aide sont prises au niveau central. D'autre 
part, la lecture originale effectuée dans cet article à partir des documents du CAPMAS se base sur notre propre lecture des annuaires d'associations des 26 gouvernorats d'Égypte et est confirmée par la plupart des informations et études réalisées à ce sujet.

51 Premier constat : les associations concernées font montre d'un bon équilibre financier, signe d'une bonne gestion, de la réussite de leurs activités et de leur attractivité aussi bien auprès $d u$ public utilisateur qu'auprès des donateurs. Les deux derniers documents du CAPMAS montrent de manière continue que les associations qui gèrent plusieurs activités sont celles qui disposent du budget le plus important. Ainsi en 1988, les 241 associations de ce type disposent d'un budget total de 28.314.156 LE, suivies par les 259 associations d'aide sociale avec 17.696.619 LE.

Second constat : la part provenant de leurs fonds propres et obtenue grâce aux services et produits qu'elles proposent ou aux revenus de leurs propriétés constitue $35 \% \mathrm{du}$ total de leurs ressources. Quant aux ressources qui manifestent leur attractivité auprès des donateurs, elles en constituent plus de $14 \%$.

53 Il importe donc de nuancer la vision caricaturale qui a généralement cours sur le financement du mouvement caritatif islamique. La réussite des activités sociales de ces associations est peut-être un des éléments principaux de leur équilibre financier. Il importe également d'insister sur le fait que les donateurs privés n'accordent leurs subventions qu'aux associations en lesquelles ils ont confiance et qui prouvent leur efficacité sur le terrain.

La réussite de ces associations vient également de leur utilisation judicieuse du dispositif juridique égyptien : elles savent en effet évoluer entre ce qui est permis et ce qui est interdit par la loi sur les associations, le code des impôts et des douanes, le droit du travail, etc. Ainsi, l'État égyptien n'intervient pas dans la construction d'une mosquée alors qu'il intervient - en imposant de multiples demandes d'autorisations dans la construction d'une école ou d'un atelier de tissage; or une mosquée, outre le fait qu'elle draine des donations importantes sans contrôle administratif préalable ou postérieur, peut également servir immédiatement d'école, de salle de cérémonies, d'atelier de couture... sans souffrir des blocages administratifs habituels.

55 À partir de ces constats, corroborés par la dernière étude ${ }^{48}$ effectuée sur la question, on peut, pour finir, s'interroger sur les relations entre l'État égyptien et ces associations à travers la question de leur financement.

\section{L'État égyptien et les services sociaux islamiques: contrôle, concurrence et mimétisme}

56 Afin de comprendre l'attitude des pouvoirs publics égyptiens envers les associations à réfèrent islamique et, plus généralement, le bénévolat social islamique, il importe de tenir compte des éléments suivants. Il s'agit d'abord d'un phénomène ancré dans l'histoire sociale égyptienne ${ }^{49}$, révélateur d'une sorte de " réflexe social » égyptien (en dépit des théories sur la profondeur historique de l'état égyptien ou sur le centralisme politique en Égypte). Rappelons ${ }^{50}$ que les associations sociales ou caritatives sont nées bien avant la création du ministère des Affaires sociales en 1939: la première association égyptienne (c'est-à-dire composée d'autochtones) est une association à référent islamique, l'Association de bienfaisance islamique, créée en 1878, suivie en 1891 de la première association copte, l'Association de Tawfiq. 
Il faut constater également qu'au plus fort de l'interventionnisme étatique, Gamal Abdel Nasser, malgré ses rapports conflictuels avec les Frères musulmans, n'a pas cherché à interdire l'existence de ce type d'associations mais à les contrôler. Le contrôle de leurs finances a toujours été au cœur même du dispositif institutionnel mis en place par les pouvoirs publics pour éviter que la vie associative égyptienne ne leur échappe. La création de la Caisse d'aide aux associations et aux fondations, en 1964, est venue parfaire ce contrôle : un de ses principaux objectifs était de drainer vers elle les sommes versées par les banques et les sociétés commerciales au profit d'oeuvres sociales en application de la loi de 1954.

Le fait que, jusqu'en 1983, la Caisse n'est pas parvenue à attirer ce genre de donations est significatif du peu de confiance qu'elle suscite. En fait, la plupart des donateurs préfèrent choisir les associations qu'ils aident pour contrôler l'utilisation de leurs subventions. Les associations à réfèrent musulman, comme on l'a vu plus haut, attirent les donations de riches hommes d'affaires, qui en font leur zakat, et celles des travailleurs égyptiens à l'étranger, notamment dans les pays du Golfe. De l'avis même des responsables administratifs ${ }^{51}$, la suppression, en 1986, des exonérations douanières sur les fonds en provenance de l'étranger et à destination du secteur associatif a remis en cause une importante source de financement de ce secteur, ce qui montre l'importance du volume de fonds en provenance des pays du Golfe ainsi que la crainte des autorités égyptiennes devant ce phénomène qui, à terme, risquait de remettre en cause l'équilibre des forces entre le ministère et les associations "à référent islamique » ou du moins une partie d'entre elles ${ }^{52}$. L'année 1986 semble être une année charnière à ce propos.

L'utilisation d'une partie du réseau associatif par le courant politique islamique dans ses différentes tendances est une réalité indéniable, même s'il est erroné d'établir un lien organique entre celui-ci et l'ensemble du réseau associatif musulman. Dans un article du journal al-Gumhuriyya du 20 janvier 1991, Mustapha Kamal al-Saïd estime très justement qu'une grande majorité de ces associations accomplissent une mission religieuse authentique, dépourvue d'arrière-pensées politiques ou d'intérêts économiques. D'autres sont liées à ce qu'on appelle l'islam politique. Certaines sont liées aux Frères musulmans - ce sont les plus modérées et les plus tolérantes certaines le sont aux extrémistes et servent de couvertures à d'autres activités. Le réseau associatif contrôlé par le courant politique islamique peut concerner plus d'une centaine d'associations puisque, lors de la rencontre historique entre le président Sadate et les représentants du mouvement islamiste, juste avant son assassinat, environ une centaine de présidents d'associations politiquement influentes étaient invités ${ }^{53}$. Il est probable que ces associations ont maintenu leurs activités, peut-être sous d'autres noms, sous la présidence de Hosni Moubarak, et les décisions administratives de 1986 supprimant les exonérations douanières ainsi que la réduction de $50 \%$ du prix de leur consommation en gaz et électricité sont notamment liées aux risques de rupture de l'équilibre dont il a été question plus haut.

60 En fait, l'attitude des pouvoirs publics égyptiens à ce propos n'est pas monolithique. Les pôles administratifs décisionnels se partagent entre le ministère des Affaires sociales, qui dispose des pouvoirs les plus importants sur la vie associative (création, suppression et contrôle financier de l'association), le ministère des Waqfs qui, malgré son influence moindre sur la vie associative à réfèrent islamique, a toutefois voix au chapitre dans un domaine qui le concerne en premier lieu, et enfin - peut-être 
surtout - la Banque Nasser Ijtimâ'i qui, elle, dispose véritablement de moyens humains et matériels pour ses activités caritatives.

61 La politique du ministère des Affaires sociales et plus largement des pouvoirs publics égyptiens envers ce type d'associations apparait en réalité tiraillée entre deux exigences contradictoires : d'une part, laisser agir ce type d'associations, dont tous les responsables administratifs ne peuvent pas ne pas admettre la réussite sur le terrain, notamment grâce à la multiplicité des services qu'elles rendent à leur environnement et à leur proximité des besoins sociaux dans un contexte de désengagement de l'État ; d'autre part, veiller à ce qu'elles ne prennent pas trop d'importance dans la vie sociale de certains quartiers, ce qui rendrait difficile l'intervention des pouvoirs publics s'il s'avérait qu'elles sont à l'initiative de cette tendance du courant islamique avec lequel il n'y pas de consensus possible, même implicite.

La politique de l'État égyptien envers le courant politique islamique se reflète dans son attitude envers les associations à réfèrent islamique : combattre la tendance " dure ", soit par la dissolution des conseils d'administration, soit par la dissolution de l'association elle-même, et collaborer avec la tendance "modérée » ou l'imiter. Cette volonté de collaboration et de mimétisme s'est dernièrement manifestée dans les dernières déclarations du ministre des Waqfs rapportées par le journal Al-Ahrâm du 22 février 1993 : «Il n'est pas dans l'intention de l'État de supprimer les associations religieuses ${ }^{54}$, dit-il ; l'État veut collaborer avec elles dans le domaine de la prédication ; ainsi, pour la première fois, elles vont participer aux caravanes de prédicateurs que le ministère organise pendant le mois du Ramadan. » De même, le ministère annonce la création de mille mosquées-cathédrales, donc de complexes sociaux autour de ces mosquées.

L'annonce de ce type de mesures n'est pas nouveau. Cela montre que les pouvoirs publics égyptiens n'entendent pas laisser les Frères musulmans occuper le terrain ${ }^{55}$. Ainsi, devant l'efficacité dont ont fait preuve, après le tremblement de terre d'octobre 1992, les institutions de la société civile contrôlées par le courant islamique ${ }^{56}$, le ministre des Waqfs ${ }^{57}$ annonce, lors d'une réunion avec les prédicateurs du gouvernorat de Giza, que la nouvelle politique du ministère pour l'année 1993 sera la suivante : 300 mosquées vont être transformées en jamà'a, c'est à dire en complexe comprenant une école coranique, un dispensaire, une bibliothèque, un atelier; chaque village bénéficiera d'un tel complexe et 20 millions de LE ont été débloquées à ce propos. De même, la décision adoptée, après le tremblement de terre, d'imposer aux donateurs de verser leurs oboles exclusivement à l'Association du Croissant rouge montre la lutte acerbe que mènent les pouvoirs publics pour s'imposer sur le terrain de la solidarité sociale, fortement concurrencés depuis plus d'une décennie par les associations religieuses musulmanes.

Cette attitude de mimétisme n'est pas nouvelle de la part de l'État égyptien, et ses outils principaux sont les comités locaux de la zakat de la Banque Nasser Ijtimâi et les associations islamiques proches des autorités publiques, auxquelles l'État accorde toutes les facilités (cf. le don de terrain à l'Association médicale islamique). Un des objectifs de ces dons est de drainer vers les associations proches des pouvoirs publics ce qu'on peut appeler l'argent islamique ». Autre exemple de cette même stratégie: les associations de développement social vivement encouragées par le ministère des Affaires sociales à cause de leurs activités économiques et qui affichent, parmi leurs multiples activités, l'apprentissage du Coran... 


\section{ANNEXES}

\section{Un exemple de « ahl al-khayi »}

La médiation étatique n'est pas la seule à souffrir du manque de confiance des donateurs égyptiens. La médiation associative peut également engendrer la méfiance. La pratique caritative, décrite ci-après, d'un riche homme d'affaires alexandrin en est une illustration.

N. A., 37 ans, marié mais sans enfants, dirige avec son frère une entreprise familiale de représentation d'outils mécaniques et électriques pour le compte de sociétés étrangères. Pratiquant la charité pour accomplir la zakat, il appartient à cette catégorie de personnes communément désignées par l'expression ahl al-khayr, employée couramment dans la presse égyptienne et qui correspond peu ou prou à l'expression française " gens de bien ", ou personnes qui pratiquent telle ou telle forme de bienfaisance (dons en argent ou en nature, terrains, immeubles...). Leur particularité est de tenir à l'anonymat, 'valeur ajoutée' à la charité islamique - ne pas se glorifier de ses bonnes actions ni chercher à en tirer un bénéfice quelconque, notamment en terme de prestige et de publicité.

C'est bien le cas de ce riche homme d'affaires alexandrin, qui nous a fait promettre de ne pas dévoiler son nom mais s'est montré très coopératif en nous accordant un entretien et en nous permettant de consulter les dossiers relatifs à sa pratique caritative, véritable petite administration avec un budget relativement important et deux employés à temps plein - un responsable, ancien fonctionnaire en retraite du ministère des Affaires sociales, et son assistant, chargé d'aider les handicapés moteurs. Ils reçoivent environ 2.000 demandeurs le 5 de chaque mois. Ces derniers (parmi lesquels beaucoup de veuves et de divorcées) présentent généralement les papiers administratifs ou médicaux attestant de leur situation. En cas de doute, les deux gestionnaires mènent une enquête. Une fois adopté le principe d'aide, chaque ayant droit reçoit une carte à deux volets recto/verso, numérotée et de couleurs différentes selon le sexe du bénéficiaire. Sur le premier volet figure, après une invocation à Dieu, la date à laquelle a commencé la distribution de la zakat du Hajj X et de ses enfants (ici, le 5/5/1985), le nom de l'ayant droit, son adresse, sa signature et sa photo, ainsi qu'un tableau énumérant les 12 mois de l'année, de 1985 à 1995.

$\mathrm{Au}$ dos de la carte, une autre invocation à Dieu et un verset coranique, puis le nom de la société, l'adresse, le numéro de téléphone et la mention suivante :

Cette carte de la zakat est distribuée aux orphelins et aux pauvres le 5 de chaque mois. Nous distribuons également des chaises et des cannes pour handicapés après étude de la situation sociale du demandeur, cela depuis le 5/5/1985. Tous les vendredis, avec l'aide de Dieu, nous offrons des soins naturels gratuits pour toute maladie rhumatismale (jambe, genou, colonne vertébrale). Nous offrons les mêmes soins naturels, sans faire usage de plâtre ni d'aucun pansement contraignant, tous les jeudis au Caire, au 15 rue... En quelques minutes on te donnera l'eau et l'huile bénéfiques que tu apposeras sur toute douleur, et Dieu te guérira. 
Cette carte a été instituée par la grâce de Dieu pendant l'ère de Moubarak - que Dieu le protège, lui, sa famille, son peuple ainsi que les dirigeants de l'Égypte et leurs alliés [...]. Qu'il éloigne d'eux tous les maux, maladies et envie. Qu'il leur accorde le bien, la pureté et l'amour et que les peuples soient protégés de toutes calamités par l'amour et la pureté.

Le tableau de distribution de la zakat du mois de janvier 1993 montre plusieurs catégories d'aides financières, qui s'échelonnent entre 2,5 LE et $40 \mathrm{LE}$. Le total représente une somme de 7.156 LE. Hormis ces aides régulières, les mandats envoyés aux nécessiteux sont doublés pendant les fêtes religieuses (environ six par an). La direction des affaires sociales d'Alexandrie envoie également les cas d'urgence à N. A. Celui-ci fournit encore gratuitement des outils de production aux ateliers de « familles productives ", programme d'aide sociale mis en place par le ministère des Affaires sociales.

Autre forme de la pratique caritative de N. A. : le financement pendant deux ou trois jours, lors des différents mûsem (fêtes de saints), de consultations médicales pour les pauvres, données par un de ses amis médecin qui, avant la date prévue, distribue un prospectus dans le quartier où il pratique : « Le docteur Untel souhaite bonne fête aux habitants de... à l'occasion de la nuit de Usraet de Mihrag - que Dieu leur accorde biens et bienfaits - et leur annonce qu'à l'occasion de cette journée bénie, des consultations et des soins gratuits seront offerts à son dispensaire les trois jours suivants. »

N. A. fait ainsi « d'une pierre deux coups » : d'une part, il permet à un nombre important de nécessiteux de se soigner gratuitement pendant trois jours, d'autre part, il attire de la clientèle à son ami médecin, qui a du mal à faire démarrer son nouveau cabinet. N. A. projette également de créer un complexe islamique sur le terrain qu'il a acheté dans son quartier d'origine. La mosquée est déjà en construction, les plans du complexe - dont les éléments centraux seront une clinique et une pharmacie - sont en cours de réalisation.

Notons pour finir que malgré l'importance de sa pratique caritative, N. A n'entend pas se constituer en association car, dit-il, « financièrement, je n'ai besoin ni du ministère des affaires sociales ni de quiconque. Je préfère être libre de mes actes et n'avoir à répondre à aucun contrôle ${ }^{58}$ ".

\section{NOTES}

1. Union générale des associations et fondations privées, Étude de la politique de l'action sociale privée en Égypte, 1983, Le Caire.

2. IMAM YOUSSEF Ibrahim, «Le financement de l'action sociale privée » in Étude de la politique de l'action sociale privée en Égypte, op. cit. p. 35 à 49.

3. Selon lui, les dons internes à l'Égypte ont également beaucoup augmenté, la politique d'infitah (ouverture économique) ayant redonné confiance aux citoyens dans les projets sociaux privés.

4. À titre d'exemple, les derniers rapports du CAPMAS sur les associations subventionnées par l'État donnent des informations datant de trois ou quatre ans avant leur parution.

5. Lire notre article, «l'État égyptien et le monde associatif à travers les textes juridiques (introduction et problématique) ", in Égypte/Monde arabe $\mathrm{n}^{\circ}$ 8, $4^{\mathrm{e}}$ trimestre 1991, Le Caire.

6. Environ $80 \%$ selon les chiffres de 1982 (in Étude de la politique... op. cit.).

7. En 1981/82, la somme obtenue s'élevait à 726.251 LE (in Étude de la politique... op. cit.). 
8. En 1981/82, la somme déduite du budget du MAS s'élevait à 8.249.788 LE, et à 407.081 LE celle déduite des budgets des autres ministères (ibid.).

9. En 1981/82, 86.852 LE (ibid.).

10. En $1981 / 82,659.918$ LE (ibid.).

11. Ibid.

12. 'ABD AL-RAHMAN Assad, «Les problèmes du financement de l'action privée arabe et l'exemple de la fondation 'Abd al-Hamid Shuman ", in Congrès des organisations privées arabes, Le Caire, 11 octobre-3 novembre 1989.

13. IMAM YOUSSEF Ibrahim, op. cit.

14. Ce genre d'associations remplit une fonction sociale très importante. Le ministère des Affaires sociales a souhaité, à un moment donné, intervenir dans le fonctionnement des petites associations d'originaires de telle ou telle région en les faisant fusionner, mais a rencontré une vive résistance du fait de la fonction de protection sociale qu'elles remplissent et de leur type d'organisation, qui permet à tous les membres de contrôler l'utilisation de l'argent mis en commun pour financer leurs différents besoins.

15. Cf. notre article « l'État égyptien et le monde associatif... », op. cit.

16. En 1977, il n'a été distribué que 52.080 LE alors que le montant du crédit était de $800.000 \mathrm{LE}$ (voir IMAM YOUSSEF Ibrahim, op. cit.).

17. Ford Fondation/Ministère des Affaires sociales, Les causes du succès et de l'échec des organisations volontaires en Égypte, Le Caire, 1990.

18. Voir notre article «Zakat officielle et zakat non officielle aujourd'hui en Égypte ", in Égypte/ Monde arabe $\mathrm{n}^{\circ} 7,3^{\mathrm{e}}$ trimestre 1991, Le Caire.

19. Ibid.

20. À titre d'exemple, l'Association de Sidi Gaber et l'Association de Sidi Bichr à Alexandrie (ces informations sont le fruit d'enquêtes de terrain menées à Alexandrie en janvier 1993).

21. Al-Gumhuriya du 20 juin 1986.

22. Al-Akhbar du 10 février 1988.

23. Al-Akhbar du 3 janvier 1987.

24. L'État n'est pas le seul à souffrir d'un manque de confiance de la part des donateurs. Le secteur associatif en pâtit également, aussi nombre de personnes préfèrent gérer elles-mêmes leurs pratiques caritatives (voir à ce propos l'annexe à cet article).

25. Le décret d'application de la loi $\mathrm{n}^{\circ} 32$ de 1964 prévoit les domaines d'activités suivants: assistance à la mère et à l'enfant, à la vieillesse, à des catégories spéciales de la population et aux handicapés, services culturels, religieux et scientifiques, développement social, organisation administrative, aide aux prisonniers et à leurs familles, planning familial, amitié entre le peuple égyptien et les autres peuples, aide aux retraités, activités littéraires.

26. AL-MAHI Mustapha, IMAM YOUSSEF Ibrahim, «Les domaines d'activités de l'action sociale privée » in Etude de la politique de l'action sociale privée, op. cit. p. 18 à 34.

27. CAPMAS, Statistiques des associations de charité subventionnées en 1986, Le Caire, août 1990.

28. CAPMAS, Statistiques des associations de charité..., op. cit., ainsi que Statistiques des associations... en 1987, Le Caire, janvier 1991, et Statistiques des associations... en 1988, Le Caire, mai 1992.

29. Rapport annuel 1990 du ministère des Affaires sociales.

30. Dans la mesure ou le répertoire des associations en Égypte montre le faible nombre d'associations culturelles et scientifiques, on peut considérer que la quasi-totalité de cette catégorie sont des associations à réfèrent religieux, plus précisément islamique.

31. Ford Fondation/Ministère des Affaires sociales, Les causes du succès et de l'échec... op. cit.

32. Document non publié.

33. En ce qui concerne le nombre exact d'associations à réfèrent islamique en Égypte, il apparaît une divergence entre les données du ministère des Affaires sociales et celles de la presse. Pour notre part, nous préférons nous en tenir au chiffre de 11.360 en nous basant sur les répertoires 
existant dans les 26 gouvernorats d'Égypte, chiffre qui correspond à peu près aux dernières données fournies par l'administration générale des associations et des unions dans un document non publié intitulé : Données sur les associations déclarées en conformité avec la loi $n^{\circ} 32$ de 1964, 1992. Pour plus d'informations sur cette question controversée, voir notre article «Le mouvement associatif égyptien et l'Islam », in Maghreb-Machrek n 135 (janvier-marsº1992).

34. Voir notre article sur la zakat, op. cit.

35. Al-Liwa' al-lslâmi du 19 octobre 1989.

36. En 1882/83, le ministère notait que cette source de financement avait accusé une baisse, alors que cela était en réalité un signe de la réussite des activités des associations et leurs bonnes relations avec leur environnement (in Étude de la politique de l'action sociale privée, op. cit.).

37. La loi $n^{\circ} 32$ de 1964 permet aux associations d'œuvrer dans plusieurs domaines d'activités après autorisation de l'administration compétente.

38. Étude de la politique de l'action sociale privée..., op. cit., p. ${ }^{\circ} 48$.

39. Ford Fondation/Ministère des Affaires sociales, Les causes du succès et de l'échec..., op. cit.

40. Étude de la politique de l'action sociale privée..., op. cit., p. ${ }^{\circ} 47$.

41. CAPMAS, Labour Information System Project, Results of the labour force sample survey fourth quarter 1988,December 1990.

42. Conseil national de la mère et de l'enfance, Rapport de préparation du troisième plan quinquennal 1992-1997.

43. Informations recueillies en janvier ${ }^{\circ} 1993$ auprès de médecins travaillant pour des associations installées à Alexandrie.

44. Lire également à ce propos l'article de Gihad 'AUDA, «Les valeurs et la culture dominantes comme critère d'évaluation des politiques publiques: étude de cas sur quelques actions islamiques ${ }^{\circ}$ ", in Evaluation des politiques publiques. Centre d'études politiques de l'université du Caire, Le Caire, 1989 (ouvrage collectif en arabe).

45. Lire à ce propos : «Les difficultés financières des associations à la lumière des dernières lois et décisions administratives ${ }^{\circ} »$, Ford Fondation/Ministère des Affaires sociales, Les causes du succès et de l'échec... op. cit.

46. Al-Gumhuriya du 20 janvier 1991 : «Les associations religieuses font de la politique malgré l'interdiction de la loi. ${ }^{\circ} \gg$

47. Ford Fondation/Ministère des Affaires sociales, Les causes du succès et de l'échec... op. cit.

48. Ibid.

49. Il est intéressant de noter que Ihya Hasan Darwich, important fonctionnaire des affaires sociales et responsable du secteur des associations, lie le mouvement associatif égyptien à la civilisation et à la religion musulmanes. A son avis, ces associations sont les héritières de grandes institutions islamiques, celles du waqf, du muhtasib et évidemment de la zakat (cf. «L'Histoire de l'action sociale volontaire dans la société égyptienne ${ }^{\circ}{ }^{\prime}$, in Congrès des organisations privées arabes, Le Caire, op. cit.).

50. Cf. notre article, «l'État égyptien et le monde associatif... », op. cit.

51. Ford Fondation/Ministère des Affaires sociales, Les causes du succès et de l'échec... op. cit.

52. Al-Gumhuriya du 20 janvier 1991, op. cit.

53. Ibidem.

54. Cette mise au point du ministre des Waqfs peut s'expliquer par le fait qu'une certaine presse, au mois de juillet 1992, a violemment attaqué les associations religieuses d'Égypte en les accusant de ne rien faire pour combattre le terrorisme, campagne qui s'est particulièrement accentuée au moment du tremblement du terre.

55. Lire à ce propos l'article de Gihad 'AUDA, op. cit.

56. Al-Wafd du23 octobre 1992, "Les syndicats et le tremblements de terre ${ }^{\circ}$ ».

57. Al-Ahrâm du 30 décembre 1992. 
58. N. A. aurait pourtant tout intérêt à se constituer en association dans la mesure où le code des impôts accepte de déduire de l'assiette imposable des revenus des personnes physiques ou des sociétés les dons versés au profit d'associations.

INDEX

Mots-clés : services sociaux islamiques, zakât

\section{AUTEUR}

SARAH BEN NÉFISSA

Cedej-Orstom 